\title{
Daseinsvorsorge und Öffentliche Mobilität: Die Rolle des Gewährleistungsstaats
}

\section{Oliver Schwedes und Roman Ringwald}

\section{Einleitung}

Der öffentliche Verkehr wird seit Beginn der Bundesrepublik als Aufgabe der Daseinsvorsorge verstanden. Eine Reform des öffentlichen Verkehrs im Sinne unseres Verständnisses von Öffentlicher Mobilität (siehe den Beitrag von Wolking in diesem Band) erfordert daher auch eine zeitgemäße Weiterentwicklung des Konzepts Daseinsvorsorge. Dazu ist es notwendig, ein Verständnis für die gesellschaftliche Funktion dieses verwaltungswissenschaftlichen Begriffs zu erarbeiten. Begriffsgeschichtlich eine relativ junge Konstruktion, hat die gesellschaftspolitische Idee der Daseinsvorsorge eine lange Tradition, die bis ins 17. Jh. zurückreicht. Die Entstehung des Prinzips der Daseinsvorsorge ist auf das Engste mit der Herausbildung des modernen Wohlfahrtsstaats verknüpft (Ritter 1991). ${ }^{1}$ Sie ist Teil einer Entwicklung, in deren Verlauf sich die Vorstellung von einem gelungenen Leben immer auch mit dem Wunsch nach Freiheit von materieller

\footnotetext{
${ }^{1}$ Während Ritter den Begriff des Sozialstaats wählt, verwenden wir den internationalen Terminus Wohlfahrtsstaat (,welfare state'). In dieser begrifflichen Trennung steht der Sozialstaat für punktuelle sozialpolitische Maßnahmen in der Tradition der Fürsorge des 19. Jh. während der wesentlich umfassendere Wohlfahrtsstaat für das erst nach dem Zweiten Weltkrieg etablierte Vorsorgeprinzip steht (auch Lessenich 2000, S. 40 f.).
}

O. Schwedes $(\bowtie)$

Technische Universität Berlin, Berlin, Deutschland

E-Mail: oliver.schwedes@tu-berlin.de

R. Ringwald

bbh Berlin, Berlin, Deutschland

E-Mail: roman.ringwald@bbh-online.de 
Not verband. Schon die Vorformen des öffentlichen Verkehrs haben sich in einer Wechselbeziehung mit denen der Daseinsvorsorge entwickelt.

Um die Herausforderung der Reform der Daseinsvorsorge für die angestrebte Öffentliche Mobilität zu erkennen, ist es hilfreich sich zunächst den geschichtlichen Werdegang zu vergegenwärtigen. In der Folge soll die aktuelle Diskussion zu dem Themenkomplex nachgezeichnet werden. Im Fazit wird die historische Perspektive auf die beiden miteinander verknüpften sozialen Aspekte beibehalten, um den politischen Charakter der Verbindung anhand von verschiedenen Handlungsalternativen deutlich zu machen.

\section{Die historische Entwicklung von Daseinsvorsorge und Verkehr}

\subsection{Die frühbürgerliche Phase}

Mit den seit dem 15. Jh. aufgenommenen Handelsbeziehungen entwickelten sich neue Verbindungen zwischen den Hansestädten Europas. Auf dem Seeweg, aber auch vermehrt auf Straßen wurden Waren und Informationen ausgetauscht. Angesichts dieser wirtschaftlichen Dynamik löste sich die feudale politische Struktur mittelalterlicher Ordnung im 17. und 18. Jh. endgültig auf (Grimm 1993). Während sich in ganz Europa moderne Nationalstaaten bildeten, vollzog sich diese Entwicklung in Deutschland mit seiner Unzahl von Regionalstaaten erst viel später. Doch auch diese Staaten befreiten ihre Untertan*innen von korporativer, feudaler und kirchlicher Willkür. Dies war weniger Ausdruck der frühstaatlichen Fürsorge als vielmehr Folge wirtschaftlicher Expansion, die das Korsett des Ständestaats sprengte. Durch die zunehmend arbeitsteilige Organisation der Wirtschaft und die damit verbundene Landflucht stieg ,die Zahl der Menschen, die etwa als Tagelöhner, Heimarbeiter oder Söldner nicht mehr in die überkommenen naturalwirtschaftlichen Familien-, Gemeinde- und grundherrlichen Verbände oder Zünfte eingegliedert waren und keine Rücklagen für Zeiten der Not und Arbeitslosigkeit machen konnten“ (Ritter 1991, S. 33). Das Ausmaß der sich ausbreitenden sozialen Krise wurde schon bald als eine Gefahr für die Gesellschaft wahrgenommen, die von der Kirche allein nicht mehr zu bewältigen war. Es entstand ein Bewusstsein dafür, dass die Gesellschaft für die Menschen, die durch soziale Missstände in prekäre Lebensverhältnisse geraten waren, eine Verantwortung habe. Diese sollte der am Gemeinwohl orientierte Staat übernehmen. In Deutschland wurde ein solcher Gedanke erstmals im Allgemeinen Preußischen Landrecht von 1794 formuliert (Koselleck 1987). Es wies die Sorge für die 
Schulen, Universitäten, Theater, die „Veranstaltung und Direktion der Volksbelustigung " (Gröttrup 1973, S. 26 f.) sowie die Organisation des Verkehrs unter die Obhut der Polizey. Sie hatte die allumfassende Aufgabe, die private Lebensführung der Bürgerinnen und Bürger zu kontrollieren und über ihre ,Glückseligkeit" sowie das physische und moralische Wohl zu wachen. Diese absolutistische Anmaßung wurde von liberalen Geistern wie Wilhelm von Humboldt stets als „drückender Despotismus“ gegeißelt (Ritter 1991, S. 43). Denn in der realen Umsetzung trat das Erbringen staatlicher Leistungen hinter den repressiven Methoden zur Herstellung sozialer Ruhe zurück. Preußen war ein Polizeistaat.

Die Interventionen des preußischen Staats im Verkehrswesen im beginnenden 19. Jh. sind in erster Linie im Zusammenhang mit militärischen Fragen zu sehen. Nach der Niederlage Preußens gegen Napoleon im Jahr 1806 wurde mit den Reformen des preußischen Beamten Freiherr vom Stein auch ein verstärktes staatliches Engagement in der Infrastruktur eingeleitet, wobei es nicht so sehr um die Unterstützung wirtschaftlicher Tätigkeit ging als darum, auf weitere kriegerische Auseinandersetzungen besser vorbereitet zu sein. Wichtig waren dabei weniger die Direktinterventionen, sondern administrative Neuerungen. So wurde mit der Städteordnung von 1808 das städtische Bürgertum gestärkt und auf die politische Verantwortung vorbereitet. In der Zeit der Restauration nach 1815 stellte sich dies als eine Aufgabenteilung zwischen Staat und Kommunen dar. Der Staat konzentrierte sich nunmehr als Nachtwächterstaat auf die innere und äußere Sicherheit, während sich die Kommunen neben genossenschaftlichen, betrieblichen und kirchlichen Organisationen um die Wohlfahrt ihrer Bürger*innen kümmerten. ${ }^{2}$ Diese gestaltete sich nach dem Fürsorgeprinzip, also der Leistung ex post für in Not geratene Menschen.

Die Aufgaben der Gemeinden wurden, vor allem nach der gescheiterten bürgerlichen Revolution von 1848, als eine Leistungsverwaltung für die bürgerliche Öffentlichkeit angesehen. Angesichts der sich jetzt auch in Deutschland abzeichnenden industriellen Revolution setzten erste systematische Überlegungen ein, wie Not und sozialen Unruhen vorgebeugt werden könnte. Es entwickelte sich eine öffentliche Daseinsvorsorge (auch wenn sie so noch nicht genannt wurde) als „Städtetechnik“ (Reulecke 1985, S. 56), also als Verfahren, um Wohnungsnot sowie schlechter sanitärer und medizinischer Versorgung $\mathrm{zu}$ begegnen. Das zu dieser Zeit aufkommende ,Gespenst des Kommunismus“

\footnotetext{
${ }^{2}$ Aus dieser Zeit rührt das Selbstverständnis deutscher Städte als korrektiver Gegenpol zum Staat. Vergegenständlicht wird dies in den Institutionen der kommunalen Selbstverwaltung.
} 
sollte mit dem ,Geist der Daseinsvorsorge“ in Gestalt der öffentlich-rechtlichen Anstalten ausgetrieben werden.

Mit der Städterechtsordnung von 1848/1849 und der Gemeindeordnung von 1850 wurde das Gemeindewirtschaftsrecht eingeführt, wodurch die öffentliche Versorgung (und die Einnahmen der Gemeinden) durch kommunale Betriebe rechtlich ermöglicht wurde. So entstanden ab 1850 Gas-, Wasser- und Abwasserwerke, erstere allerdings zunächst in privater Hand. Gemeindewirtschaftliche Interessen - das gehobene Bürgertum wollte ebenfalls von den ,neuen Märkten ‘ profitieren - sowie Unfälle, die hin und wieder bei der Gasversorgung vorkamen, als auch Masseninfektionen durch verseuchtes Wasser führten dazu, dass in fast allen deutschen Großstädten bis 1880 die Gas- und Wasserwerke kommunalisiert wurden.

Schon in der klassischen politischen Ökonomie Adam Smiths wurde darauf hingewiesen, dass eine „Zirkulation in den Kapitalen“ für eine „gesunde Ökonomie" notwendig sei (Smith 1996, S. 20 f.). Seit dem 17. Jh. wurde dieser Bedarf in den europäischen Hauptstädten durch den Betrieb von Droschken Pferdekutschen, die man für einen bestimmten Betrag und eine bestimmte Zeit mieten konnte - befriedigt. In den späten 20er Jahren des 19. Jh. traten, zunächst in französischen Großstädten, ${ }^{3} 1829$ auch in London und schließlich 1839 in Berlin Pferdeomnibusse mit festen Linien und Fahrzeiten in Erscheinung. Rechtlich geregelt wurde der Betrieb in Deutschland durch Polizeiverordnungen. ${ }^{4}$ Obwohl sich die Anzahl der Linien und Fahrzeuge schnell und stark vergrößerte, war dieses Beförderungsmittel bis zur Mitte des Jahrhunderts wegen der hohen Kosten den privilegierten Bürger*innen vorbehalten.

So lässt sich für diesen Zeitraum zusammenfassend konstatieren: Die politischen Umbrüche, vor allem der Übergang vom Absolutismus zum Verfassungsstaat, sorgten dafür, dass die Kommunen legislativ die Zuständigkeit erhielten, bestimmte Leistungen zugunsten der Bürgerinnen und Bürger zu erbringen. Durch die Industrialisierung verstärkte sich der Druck auf die Gemeinden, eine effiziente Leistungsverwaltung zu etablieren. Bis zum Ende des Jh. war der Verkehr allerdings kaum Gegenstand dieser Bemühungen.

\footnotetext{
${ }^{3}$ Nantes 1826, Bordeaux 1827, Paris 1828.

${ }^{4}$ Siehe das „Polizeireglement für den Betrieb des sogenannten Omnibus Personen Fuhrwerkes“ vom 01.02.1851, Berlin.
} 


\subsection{Die Etablierung einer Systematik in Krisenzeiten}

Erst Ende des 19. Jh. wurde der Gedanke staatlicher Vorsorge mit der Einführung der Sozialversicherungen aufgegriffen. Die gesellschaftlichen Klassenkonflikte hatten sich im Zuge der Industrialisierung soweit zugespitzt, dass der Staat mit der sozialen Sicherung „Daseinsvorsorge und Gefahrenabwehr“ (Wehler 1995, S. 1255) betreiben wollte, um gesellschaftspolitische Veränderungen einzudämmen. Zugleich wurde aber eine Sozialpolitik etabliert, die sich, im Gegensatz zur bis dahin praktizierten Armenfürsorge, durch eine neue Qualität auszeichnete: „Die neue Sozialpolitik sah einen reichsweiten, dauerhaften, durch Rechtsansprüche gesicherten effektiveren Schutz gegen Risiken des Einkommensverfalls vor" (Schmidt 2005, S. 24).

Auf kommunaler Ebene gewannen sogenannte ,munizipalsozialistische“ Ideen rasch an Einfluss. Die Protagonisten dieser aus der Finanzklassik abgeleiteten Denkrichtung ${ }^{5}$ teilten bei allen theoretischen Unterschieden die Annahme, dass ein Wirtschaftssystem, das rein privatrechtlich organisiert ist, nicht in der Lage sei, die durch die Industrialisierung entstandenen ökonomischen und sozialen Probleme zu lösen, und dass diese Funktionsdefizite durch das Eingreifen der Gebietskörperschaften zu beheben seien. Im Gegensatz zur Sozialdemokratie, die damals nach einer Überwindung der Klassengegensätze trachtete, stellten die Munizipalsozialisten als Sozialkonservative das bestehende System nicht infrage, sondern sahen gemeinwirtschaftliche Unternehmen als dessen komplementäre Ergänzung. Gleichzeitig waren sie an der Prosperität einer vom (autoritären) Staat emanzipierten Gemeinde interessiert.

Angesichts der sozioökonomischen Strukturveränderungen im Gefolge der ,explodierenden' Industriegesellschaft erfolgte eine theoretische Begründung der Notwendigkeit kommunaler Eigenbetriebe. Auch die Sozialdemokratie beurteilte die Kommunalisierung von Monopolbetrieben - im Gegensatz zur äußerst kontroversen Debatte des Gothaer Parteitags 1875 über die Verstaatlichung der preußischen Eisenbahnen - nahezu einhellig positiv, da sie meinte, über den gemeinnützigen Charakter der Unternehmen demokratische Kontrolle über sie zu erlangen und eine zielgerichtete regionale Wirtschaftspolitik betreiben zu können. 1900 waren die Kommunen schließlich nach Reichspost und Preußischer Staatsbahn die größte Arbeitgeberin im Deutschen Reich. ${ }^{5}$ Die bekanntesten Namen sind Adolph Wagner, Albert Schäffle, Gustav von Schmoller und
Emil Sax. 
Gleichzeitig mit den gemeindewirtschaftlichen Reformen wurden ab Mitte des Jh. in Großstädten verstärkt auch stadtplanerische Aktivitäten in die Wege geleitet, die einem internationalen Trend folgten. Die Namen Haussmann in Paris und Hobrecht in Berlin stehen gleichermaßen für einen an hygienischen Kriterien orientierten Städtebau mit blockmäßiger Neubebauung und breiten, lichten Straßen in rasterförmiger Anordnung. Von Anfang an beinhaltete die Stadtplanung auch verkehrsplanerische Aspekte. Für die entstehenden industriellen Großbetriebe mussten die Zuführung und der Abtransport vieler Arbeitskräfte gewährleistet werden. Für die Entwicklung einer urbanen, bürgerlichen Öffentlichkeit waren innerstädtische Verkehrswege eine notwendige Umweltbedingung.

In dieser Zeit der radikalen Umgestaltung und Ausweitung der Städte wurden in den neuen Ausfallstraßen Schienen verlegt, um mit Pferdetrambahnen die Kommunikation mit den Vororten zu beschleunigen und neue Siedlungsgebiete zu erschließen. Am 25. Juni 1865 verkehrte die erste Pferdestraßenbahn Deutschlands zwischen dem Brandenburger Tor und Charlottenburg. Aber die technischen Innovationen der Zeit ermöglichten bald ganz andere Verkehrsformen. Schon 1863 nahm in London die erste Untergrundbahn der Welt den Betrieb auf und in Berlin wurden in den 1870er Jahren Trassen für dampfgetriebene Stadteisenbahnen gebaut. Die dortige Ringbahn wurde 1877 vollendet, was zu einer „erste[n] Welle industrieller Standortverlagerungen“ (Bendikat 1997, S. 168) führte. „Diese sogenannte erste Randwanderung ließ die relativ enge Beziehung von Wohngebiet und Industriestandort in vielen Fällen zwar noch bestehen, dennoch mussten die Beschäftigten zunehmend die öffentlichen Verkehrsmittel benutzen“ (ebd.). Im Zuge dieser Entwicklung kam es allmählich zur ,Veröffentlichung ' des privaten Nahverkehrs. In zäher Auseinandersetzung mit den Privateigentümern begann die öffentliche Hand um die Jahrhundertwende Tarife durchzusetzen, die auch für Arbeiter*innen erschwinglich waren. ${ }^{6}$ „Gerade die Feststellung, dass öffentliche Nahverkehrssysteme historisch für eine zunächst nur schmale Zielgruppe wohlhabender Benutzer entstand, wie Linienführung,

\footnotetext{
${ }^{6}$ In Berlin wurde 1910 der 10-Pfennig-Einheitstarif für alle Straßenbahnen eingeführt. Nicht zuletzt dadurch konnte die Straßenbahn ihre Fahrgastzahlen im Dekaden-Rhythmus verdoppeln und wurde zum wichtigsten Verkehrsmittel des sich ausweitenden Berufsverkehrs (Wolf 1992, S. 33, 39). Dieser Fahrpreis konnte jedoch nur aufgrund massiven politischen Drucks (und nach dreijährigen Verhandlungen zwischen Betreibern und Stadtverwaltung) eingeführt werden. Die Straßenbahngesellschaften favorisierten aus Profitinteressen ein Festhalten an höheren Preisen (Bendikat 1997, S. 171).
} 
Verkehrsdichte und Kapazität verdeutlichen, lässt schließlich die Tarife als wichtige Indikatoren für den Wandel in den Zielgruppen der öffentlichen Nahverkehrsmittel erscheinen" (Fisch 1997, S. 57). Doch obwohl sich die Kommunen mit öffentlichen Subventionen engagierten, um sozial verträgliche Fahrpreise zu gewährleisten, blieb der politische Einfluss auf die Gestaltung des Personennahverkehrs zunächst gering.

Die von Unternehmen wie Siemens und AEG durchgeführte Elektrifizierung der Straßenbahn - die erste elektrische Tram der Welt fuhr 1881 im Berliner Vorort Lichterfelde - trieb die Industrie- und Verkehrsentwicklung weiter voran. Im Engagement dieser expandierenden Großbetriebe erwiesen sich die neuen Nahverkehrstechniken als wesentliche Triebkräfte. Einerseits stellten sie die verkehrliche Infrastruktur für die Ausweitung der gesamten industriellen Produktion, andererseits bedeutete dies für die beteiligten Firmen ein immenses Wachstum. Als Folge dieser Dynamik entwickelte sich der öffentliche Verkehr zum Massenverkehr, wobei die Kommunen am Ende des 19. Jh. begannen, Monopolisierungsbestrebungen im Nahverkehr zu unterstützen oder sogar anzuordnen. ${ }^{7}$ Damit intendierten sie darauf, dass die einzelnen, unabhängigen und zum Teil aneinander vorbeifahrenden Linien zu einem System zusammenwuchsen, welches den Anforderungen der expandierenden Großstädte gerecht wurde. Zunächst handelte es sich je nach Verkehrsträger um (zumeist private) Monopolgesellschaften. So gab es um 1900 in Berlin zwar eine monopolisierte Allgemeine Berliner Omnibus AG (ABOAG), daneben aber viele verschiedene Straßenbahnbetreiber, unter ihnen als weitaus größte die Große Berliner Straßenbahngesellschaft sowie ab 1902 die von Siemens und Deutscher Bank gegründete Gesellschaft für elektrische Hoch- und Untergrundbahnen in Berlin.

Landesweit machten städtebauliche Veränderungen und die Notwendigkeit von Grundstücksenteignungen für U-, S- und Straßenbahnbau schon in der Initialphase eine Kooperation zwischen privaten Investoren und planerischer Instanz erforderlich. Mit den immer noch steigenden Bevölkerungszahlen in den Städten setzte sich in den Jahren um 1900 eine Doktrin verstärkter kommunaler Investitionen und Vorsorge in den Verwaltungen durch. Die Kommunalisierung des urbanen Verkehrs erfolgte im Vergleich zur Wasser-, Gas- und Elektrizitätsversorgung

\footnotetext{
${ }^{7}$ In Berlin übernahm die 1865 neugegründete Berliner Omnibus-Gesellschaft per Polizeidekret die Konzessionen sämtlicher bisheriger (Pferde-)Omnibus-Unternehmer. 1898 förderte die Stadt Berlin den Zusammenschluss dreier Straßenbahnbetriebe zur Großen Berliner Straßenbahngesellschaft (GBS) durch großzügige Subventionierung von Neubauten, Elektrifizierung und die Verlängerung der Konzession (Bendikat 1997, S. 70).
} 
relativ spät - meistens nach der Jahrhundertwende - und schleppend. Einerseits waren viele private Gesellschaften nicht bereit, das profitable Geschäft aus der Hand zu geben. Andererseits entsprach ihre an der Rentabilitätslogik orientierte Investitions- und Expansionspolitik nicht den Erwartungen der Kommunalpolitik, die die Erschließung von Stadträndern und die Verknüpfung der verschiedenen Strecken forcieren wollte.

Die Versorgung mit Nahverkehr durch öffentlich-rechtliche Anstalten wurde bis zum Krieg nur teilweise verwirklicht. Die munizipalsozialistischen Ideen, die dies beförderten, setzten sich in den Verwaltungen allerdings mehr und mehr durch. Der Gegensatz von gemeinwohlorientierter Verwaltungstätigkeit und privatwirtschaftlichem Profitstreben wurde mit der Einführung des investitionsintensiven, elektrifizierten Massenverkehrs immer deutlicher (Wysocki 1995). Gleichwohl lässt sich für diese Zeit ein für den öffentlichen Nahverkehr förderliches Zusammenspiel aus staatlicher Regulierung und privatwirtschaftlicher Initiative konstatieren.

\subsection{Gemeinwirtschaft und (Im-)Mobilisierung im Krieg}

„Die Mobilisierung der Gesellschaft" (Lederer 1979, S. 120) im Ersten Weltkrieg forcierte die Staatstätigkeit in der Wirtschaft. Auch auf kommunaler Ebene wurden die unter dem Kriegsprimat stehenden Konzepte einer ,Gemeinwirtschaft" umgesetzt, in der staatlich monopolisiert und konzessioniert produziert wurde. Dies beinhaltete auch sozialpolitische Zugeständnisse an die Arbeiter*innen. ${ }^{8}$ Indessen führte das Vaterländische Hilfsdienstgesetz mit der Zwangsverpflichtung, den eingenommenen kriegswichtigen Arbeitsplatz zu behalten, zur ,Immobilisierung ' der Arbeiterinnen und Arbeiter. Das zeigte sich auch im von Mangelwirtschaft eingeschränkten öffentlichen Verkehr. Eisen, Stahl, Kohle und Benzin wurden in erster Linie für die unmittelbare Kriegsproduktion verwendet. Selbst die in der korporativen Kriegsökonomie herausragende Stellung Walter Rathenaus, der als Vorstand der AEG ein Unternehmen repräsentierte, das an der Nahverkehrstechnik glänzend verdiente, konnte den

\footnotetext{
${ }^{8}$ Deshalb wird diese Zeit auch als ,,die entscheidende Phase bei der Umwandlung der alten, nie rein existierenden Ordnungsverwaltung zur Leistungsverwaltung des modernen Sozialstaats“ bezeichnet (Kocka 1999, S. 121).
} 
Zusammenbruch des Nahverkehrsmarkts nicht verhindern. Omnibusse im Stadtverkehr wurden sofort nach Kriegsbeginn vollständig für militärische Zwecke requiriert. Die dampfbetriebenen Nahverkehrsbahnen wurden stark reduziert, Straßenbahnen eingeschränkt betrieben. Die noch arbeitenden Anlagen wurden voll auf Verschleiß gefahren. So führte die korporative Kriegswirtschaft schließlich zur Epoche der Kommunalisierung. Denn nach dem Krieg waren viele Nahverkehrsunternehmen schlichtweg bankrott und die Kompensation des Verschleißbetriebs machte hohe Investitionen notwendig. Die Verkehrsträger waren heruntergewirtschaftet und mussten nun mit staatlichen Geldern saniert werden. In den meisten Fällen übernahmen die Kommunen die Betriebe und vollzogen damit eine Privatisierung der Gewinne und die Verstaatlichung der Verluste (Wolf 1992, S. 48).

\subsection{Staatsintervention als Zeitzeichen der Weimarer Republik}

Die allgemeine Ausweitung der staatlichen Eingriffe in die Wirtschaft der Weimarer Zeit ist geprägt durch das Scheitern der Revolution 1918/19, eine gleichwohl erstarkte reformistische Sozialdemokratie und das Versagen des Marktes. Nicht mehr rasante demographische Entwicklungen wie Ende des 19. Jh. bestimmten die Staatsinterventionen in die Wirtschaft, sondern Not und Armut infolge von Krieg und Weltwirtschaftskrise. Nachdem mit der Niederschlagung der Revolution auch eine grundsätzlich veränderte Wirtschaftsordnung nicht mehr zur Debatte stand, legte die SPD als stärkste politische Kraft der Weimarer Republik ihr Augenmerk auf die ,Demokratisierung der Wirtschaft". Durch den Ausbau der öffentlichen Betriebe und die Gründung von Genossenschaften und gewerkschaftseigenen Unternehmen wurde eine Vielzahl politischer Ziele verfolgt. So sollten insbesondere die wirtschaftlich schwachen Kommunen durch Zuschüsse aus den gegründeten Eigenbetrieben - Stadtwerke in ihrer häufigen Mischform als Energie-, Wasser- und Verkehrsversorgerin - finanziell entlastet werden. Dies gelang auch größtenteils. ${ }^{9}$

\footnotetext{
${ }^{9}$ „Über $90 \%$ der kommunalen Betriebseinnahmen stammte aus den Versorgungsbetrieben von Wasser, Gas, und Elektrizität, wobei allein die Elektrizitätswerke zwischen 30 und $50 \%$ erwirtschafteten“ (Ambrosius 1984, S. 92).
} 
Die sozialdemokratische Partei verfolgte mit den öffentlichen Unternehmen bestimmte gemeinwirtschaftliche Ziele (Ambrosius 1984, S. 60):

1. Bedarfsdeckungs- oder Versorgungsprinzip anstelle des Gewinnprinzips,

2. planwirtschaftliche Organisation anstelle konkurrenzwirtschaftlicher Beziehungen,

3. wirtschaftspolitische Instrumentalisierung anstelle ausschließlich betriebswirtschaftlicher Perspektive,

4. gemeinwirtschaftlich-demokratische Betriebsführung anstelle hierarchischer Organisationsstrukturen und

5. fortschrittliche, betriebliche Lohn- und Arbeitsbedingungen anstelle privatkapitalistischer Ausbeutung.

Wie schon im Kaiserreich trafen sich diese ,sozialistischen 'Vorstellungen mit denen eines sozialen Flügels der Liberalen. ${ }^{10}$

Aufgrund konjunktur-, raumordnungs- und sozialpolitischer Prämissen im Zusammenhang mit der Bekämpfung der Wirtschaftskrise waren sich Sozialdemokrat*innen und ,Interventionsliberale“ einig, die Investitionstätigkeit der öffentlichen Hand aller drei Gebietskörperschaften zu erhöhen. In diesem Zusammenhang wurden den öffentlichen Betrieben immer mehr Aufgaben zugewiesen. Im Bereich des öffentlichen Wohnungsbaus, der Wohlfahrt, der Sozialversicherungen, der Versorgungsbetriebe und des Verkehrswesens wurden in der Weimarer Zeit zwischen 55 und 65 Prozent aller Bruttoanlageinvestitionen getätigt (Ambrosius 1984, S. 95).

Der Ausbau des öffentlichen Verkehrs zum Massenverkehrsmittel ging einher mit einer drastischen Veränderung der Eigentumsverhältnisse: „Hielten sich vor dem Ersten Weltkrieg kommunale und private Straßenbahnunternehmen die Waage, so setzte nach dem Krieg eine systematische Kommunalisierung ein, die von einem starken Konzentrationsprozess begleitet war. Die verschiedenen Straßenbahnbetriebe, die es jeweils in den einzelnen Großstädten gab, wurden zu einem Unternehmen zusammengefasst. Zugleich fand ein umfangreicher Ausbau der Schienennetze statt" (ebd., S. 75).

In Berlin wurde nicht nur das „modernste Nahverkehrssystem Europas“ (ebd., S. 76) gebaut, es wurden auch neue Organisationsstrukturen errichtet. Schon 1921 hatte die Stadt die Mehrheit der Straßenbahnen übernommen, 1926 die

\footnotetext{
${ }^{10}$ Als herausragende Namen können hier Heinrich Niebuhr (1928), Kurt Wiedenfeld (1927), Manuel Saitzew (1930), Walter Gerber (1928) und Jürgen Brandt (1929) genannt werden.
} 
Aktienmehrheit bei der Busgesellschaft ABOAG sowie der Hoch- und Untergrundbahn. In den Berliner Verkehrsbetrieben (BVG) wurden 1929 die verschiedenen Verkehrsträger - Straßenbahn, U- und S-Bahn, Bus - zu einem integrierten Nahverkehrsunternehmen zusammengeführt. Dies war bis dahin einmalig in ganz Europa. Die Rechtsform war zunächst die der Aktiengesellschaft, an der die Stadt Berlin 100 Prozent der Aktien führte. In den 1930er Jahren wurde sie zu einer Anstalt des öffentlichen Rechts umgewandelt. Die BVG, als zu dieser Zeit drittgrößtes Unternehmen der Republik, ist das prominenteste Beispiel für die Kommunalisierung des öffentlichen Verkehrs in Deutschland der 1920er Jahre. Dort, wo es nicht schon seit dem Kaiserreich zahlreiche kommunale Eigenbetriebe gab wie in Westfalen, wurden die Verkehrsbetriebe nun massiv in die öffentliche Hand überführt. „Im Jahr 1927 betrieben 223 Straßenbahnunternehmen insgesamt eine Streckenlänge von 6.285 Kilometern. Von diesen Betrieben waren 120 rein kommunale Einrichtungen, 67 hatten gemischtwirtschaftlichen Charakter mit überwiegend kommunalem Einfluss, während sich immerhin noch 36 Betriebe in privater Hand befanden" (ebd.).

Zwar mag bei der Kommunalisierung der Verkehrsbetriebe vereinzelt der Wunsch der Gemeinden zugrunde gelegen haben, am Profit beteiligt zu werden. Doch zeigte sich darin in erster Linie die Reaktion auf ein Marktversagen (Wolf 1992, S. 55 ff.; Niederich 1997, S. 95 ff.). Die Gemeinden verfolgten mit der Kommunalisierung Gemeinwohlinteressen im Sinne dessen, was schon kurze Zeit später Daseinsvorsorge genannt wurde. Damit war ausdrücklich auch eine volkswirtschaftliche Verantwortung gemeint, da die fordistische Wirtschaft ein funktionierendes Massenverkehrssystem als quasi ,natürlich“ voraussetzte. Die ,Veröffentlichung' des urbanen Verkehrs war eingebettet in eine international zu beobachtende Ausweitung der wirtschaftlichen Staatstätigkeit (Wagner 1995). Sie war einerseits eine plausible Folge einer Machtverschiebung vom Kapital hin zur organisierten Arbeiterbewegung und andererseits eine konsequente Verantwortungsübernahme der Kommunen für das Wohl ihrer Bürgerinnen und Bürger.

Am Ende der Weimarer Republik spitzten sich die prekären sozialen Verhältnisse mit der Weltwirtschaftskrise noch einmal dramatisch zu. Vor dem Hintergrund dieses Marktversagens wurde die Idee einer starken Stellung des Staates bei der Gewährleistung existentieller Grundbedürfnisse in ganz Europa nahezu zum Common Sense (Wagner 1995). Die ,Dritte Notverordnung des Reichspräsidenten zur Sicherung von Wirtschaft und Finanzen und zur Bekämpfung politischer Ausschreitungen“ aus dem Jahr 1931 bringt diese Entwicklung exemplarisch zum Ausdruck, wobei der Titel zugleich auf den wesentlichen 
Aspekt des staatlichen Eingriffs zum Zwecke sozialer Befriedung verweist. „Insgesamt spiegeln diese Gesetze den Zeitgeist wider, der geprägt war vom Übergang vom liberalen zum interventionistischen Denken, vom föderalen zum zentralstaatlichen Politikverständnis, vom sinkenden Vertrauen in wettbewerblich strukturierte Märkte und wachsenden Glauben an die Effizienz geplanter Organisationen“ (Ambrosius 1995, S. 33). Im ,Geiste dieser Zeit" entwickelte der Staatsrechtler Ernst Forsthoff in den 1930er Jahren mit dem Prinzip der Daseinsvorsorge ein Konzept, das zum Ziel hatte, sowohl lebensnotwendige Bedürfnisse wie auch die Gesellschaft zu befrieden.

\section{Die begriffliche Fassung des Zusammenhangs von Daseinsvorsorge und öffentlichem Verkehr}

Die Daseinsvorsorge und die Diskussion um sie gab es also, bevor sie so genannt wurde. Der Begriff Daseinsvorsorge wurde 1938 von Ernst Forsthoff in einer Broschüre mit dem Titel „Die Verwaltung als Leistungsträger“ im deutschen Sprachraum eingeführt. Es ging dem Autor darum, sein Credo an eine ,neutrale“ Verwaltung zu formulieren und sie als heimliche Macht im Staat zu stärken.

Dass Daseinsvorsorge erforderlich sei, begründete der Staatsrechtler mit der im Zuge der Industrialisierung erfolgten Urbanisierung. Aus ihr erwachse eine soziale Bedürftigkeit, die dem Einzelnen die notwendigen und über das Notwendige hinausgehenden Güter nicht mehr direkt zugänglich machten. Als Beispiel nennt er „die Versorgung mit Wasser, Gas und Elektrizität“ (Forsthoff 1938, S. 7). Da Großstädter*innen keinen eigenen Hof als „,beherrschten Raum“ (Forsthoff 1938, S. 4) besitzen, müssten sie die Versorgung mit diesen Gütern im ihnen „zugänglichen Raum“ (ebd.) der Öffentlichkeit organisieren. Weder könnten die urbanisierten Menschen einen eigenen Brunnen graben, noch selbst Strom erzeugen, geschweige denn Gas herstellen. Diese Güter würden notwendigerweise von gesellschaftlichen Instanzen bezogen (Scheuner 1978, S. 547 f.). Die so definierte soziale Bedürftigkeit sei unabhängig von der ökonomischen Lage und dürfe darum mit der sozialen Fürsorge in Fällen von Armut, Krankheit oder sonstiger Not nicht gleichgesetzt werden, im Gegenteil: Fürsorge und Daseinsvorsorge müssten scharf getrennt werden.

Unter Berufung auf Max Weber verweist Forsthoff darauf, dass der moderne Mensch sich diese Güter auf bürokratisch vermitteltem Wege aneignen müsse. Der Begriff der Teilhabe steht deshalb im Zentrum der Konzeption, heute sprechen die Sozialwissenschaften von ,Access' (Zugang) (Ronellenfitsch 2003, S. 68). Auch andere prominente Gewährsleute zieht Forsthoff bei der 
Begriffsbildung heran. So beziehen sich einzelne Passagen mitunter wortwörtlich auf Friedrich Dessauers „Philosophie der Technik“ (1928), andere auf Karl Jaspers „Geistige Situation der Zeit“ (1931), in der von der Notwendigkeit der „Daseinsfürsorge“ die Rede ist (ebd., S. 31 f.). Forsthoff knüpft also an den zeitgenössischen existenzialphilosophischen Diskurs an und überträgt einen dort gebildeten Begriff auf die Theorie und Praxis der Staatsverwaltung (Meinel 2011). Zur Konkretisierung des Gegenstands verweist Forsthoff neben der Post, Telefonie und Telegraphie, der hygienischen Sicherung, der Vorsorge für Alter, Invalidität, Krankheit und Arbeitslosigkeit insbesondere auf die „Bereitstellung der Verkehrsmittel jeder Art“ (Forsthoff 1938, S. 7; Rüfner 2006, S. 1052).

Damit beschreibt Forsthoff das Verkehrswesen dezidiert als Bestandteil der Daseinsvorsorge. Ausdruck dieser Relation sei das ,Gesetz über die Beförderung von Personen zu Land“ (PBefG) vom 4. Dezember 1934. Darin werden, so Forsthoff, die Aufgaben der einzelnen Verkehrsträger durch ihre Bedeutung für den Gesamtverkehr und die Wirtschaft bestimmt. Die Subsumierung des Verkehrs unter die Daseinsvorsorge stellt sich auf diese Weise als rechtswissenschaftlicher Kommentar des PBefG dar. Der Zusammenhang von Daseinsvorsorge und Verkehr ergibt sich jedoch durch die im Prozess der Urbanisierung möglich, aber auch notwendig gewordene Mobilität, wenn diese nicht individuell zu leisten ist oder wenn sie Gemeinwohlinteressen tangiert. Unter welchen Bedingungen dieser Zusammenhang auch im Zeitalter individuell ermöglichter Massenautomobilität fortbesteht, markiert dabei die politische Diskussion um die Daseinsvorsorge unserer Tage.

\section{3 Überholtes Konzept oder bleibende Bedeutung der Daseinsvorsorge?}

Für die Frage, welche Bedeutung dem Begriff der Daseinsvorsorge - insbesondere in rechtlicher Hinsicht - heute noch zukommt, ist entscheidend, wie eng der Begriff mit dem autoritären Staatsverständnis von Forsthoff verknüpft war und ob er auf Grundlage der im Rahmen der freiheitlich-demokratischen Grundordnung der Bundesrepublik überhaupt noch sinnvoll Anwendung finden kann (Meinel 2011).

Dabei ist zu konstatieren, dass Forsthoff in seinem grundlegenden Lehrbuch zum Verwaltungsrecht den Begriff der Daseinsvorsorge zwar weiterhin verwendete, es jedoch unterließ, auf diesen Begriff eine umfassende neue Dogmatik 
des Verwaltungsrechts aufzubauen (Forsthoff 1950; Meinel 2011, S. 145). Die in seiner Schrift Die Verwaltung als Leistungsträger angelegte Idee, auf Grundlage eines funktionellen Verständnisses der Tätigkeit der staatlichen Verwaltung das gesamte Verwaltungsrecht von seiner klassischen Ausrichtung auf einzelne Rechtsinstrumente zu lösen, hat er selbst nicht umgesetzt. Im Anschluss daran ist vielfach der Einwand erhoben worden, dass der Begriff der Daseinsvorsorge zwar weiterhin von Bedeutung sein mag, jedoch eher im politischen oder soziologischen Bereich und dem Begriff eine rechtliche Qualität nicht mehr zukomme (Ronellenfitsch 2003, S. 72 f.).

Demgegenüber ist jedoch zu beachten, dass der Begriff auch weiterhin in einer Vielzahl gerichtlicher Entscheidungen Verwendung gefunden hat und beispielsweise von der Europäischen Kommission regelmäßig in Stellungnahmen zu Dienstleistungen von allgemeinem Interesse nach Art. 106 des Vertrags über die Arbeitsweise der Europäischen Union (AEUV) ganz selbstverständlich verwendet wird (ebd., S. 81 f.). ${ }^{11}$ Darüber hinaus liegen auch in der rechtswissenschaftlichen Literatur nicht wenige Veröffentlichungen vor, die dem Begriff der Daseinsvorsorge eine bleibende Bedeutung zumessen (ebd., S. 69).

Eine angemessene Bewertung zur rechtlichen Qualität des Begriffs der Daseinsvorsorge muss daran anknüpfen, dass Forsthoff mit diesem Begriff eine veränderte Verwaltungswirklichkeit aufgegriffen und juristisch verarbeitet hat (Ringwald 2008). Er knüpfte an die zunehmende Industrialisierung und die Konzentration vieler Menschen auf engem Raum in Großstädten an. Gemeint sind dabei insbesondere Bereiche kommunaler Infrastrukturen, die für die Ermöglichung eines Zusammenlebens von Menschen zwingend erforderlich sind, über die der Einzelne aber (unabhängig von seiner sozialen Bedürftigkeit) nicht selbst verfügen kann. Diese Frage bleibt aktuell und die damit verbundene Gewährleistungsverantwortung der staatlichen Verwaltung ist in fast allen kommunalen Infrastrukturbereichen heute (weitgehend) unstrittig. Landkreise und Kommunen wählen seit Jahrzehnten die Betreiber von Strom-, Gas- oder Wärmenetzen aus und sind für die Abwasserentsorgung und die Wasserversorgung verantwortlich. Sie beschränken sich dabei auch nicht auf die einmalige Auswahl, sondern treffen - im Rahmen der gesetzlichen Vorgaben - auch Entscheidungen für die Art und Weise der Leistungserbringung.

\footnotetext{
${ }^{11}$ Eine beihilferechtliche Vorgabe, die gerade im Bereich des öffentlichen Personennahverkehrs eine zentrale Rolle spielt.
} 
Besonders deutlich wird dies auch beim öffentlichen Personennahverkehr. Dessen schon rein räumlich deutlich höhere Effizienz im Vergleich zu Fahrzeugen des Motorisierten Individualverkehrs (MIV), ermöglicht in vielen Fällen überhaupt erst eine ausreichende individuelle Mobilität auf den begrenzten Verkehrsflächen. Ein reiner MIV wäre in - zumindest fast allen - bundesdeutschen Ballungszentren nicht ohne weiteres möglich und würde zu einem Kollaps vieler Verkehrsbeziehungen führen. Dies gilt ganz unabhängig davon, dass mit der aktuell besonders stark diskutierten Forderung nach einer Verkehrswende, die insbesondere eine Verlagerung vom MIV auf Verkehrsträger des Umweltverbundes adressiert, auch die Lebensqualität in Städten und der Gesundheitsschutz in der Bevölkerung nachhaltig gesteigert werden soll.

Es ist gleichwohl - mit Blick auf Forsthoff - uneingeschränkt zu konzedieren, dass die Herleitung der Daseinsvorsorge als rechtlichem Begriff eng verbunden war mit seinem autoritären Staatsverständnis (Günther 2011, S. 367). Forsthoff betrachtet den Staat als autoritäre politische Einheit, der sich als höchste rechtliche und ethische Instanz durch eine Verhinderung des Bürgerkrieges definiert und legitimiert. Insoweit knüpft Forsthoff eng an die Lehre von Carl Schmitt und seinem Verständnis des Politischen als Unterscheidung zwischen Freund und Feind an (Forsthoff 1933; Günther 2011, S. 367; Wehler 2014, S. 147). Mehr Distanz zu einer freiheitlich-demokratischen Grundordnung ist kaum denkbar (Ringwald 2008, S. 45). In dem für das Grundgesetz so wesentlichen Wettstreit zwischen verschiedenen Meinungen vermag Forsthoff nicht mehr als die stets virulente Gefahr bürgerkriegsähnlicher Zustände zu erkennen.

Auf Grundlage dieser Überlegungen beschreibt er die Legitimation für ein rechtlich ungebundenes Sachermessen der Verwaltung. Anstelle der normativen Bindung tritt die Bindung an einen bestimmten Sachzweck. Grenzen sind im Verwaltungshandeln nur bei ausschließlich subjektiven und sachfremden Erwägungen geboten. Seine Verwaltungsrechtslehre der Daseinsvorsorge dient also dazu, die Verwaltung gegenüber der parlamentarischen normativen Sphäre soweit wie möglich zu verselbstständigen und unmittelbar zum Instrument einer parlamentsunabhängigen Staatsführung zu machen. Dies steht im starken Kontrast zu den modernen Ansprüchen einer normativen Integration der administrativen Planung (siehe dazu Beitrag von Schwedes in diesem Band).

Jede Kritik am Begriff der Daseinsvorsorge, die diesen Hintergrund seiner Entwicklung aufgreift, ist daher auch unbedingt berechtigt (Knauff 2004, S. $43 \mathrm{f}$.). Es ist auf Grundlage der unveräußerlichen Menschenwürde als Fundamentalnorm des Grundgesetzes nicht möglich, jede staatliche Entscheidung für konkret verbindlich oder metaphysisch gerecht zu erklären. Der Staat ist keine 
vorrechtliche Größe, sondern die Staatsgewalt geht vom Volke aus und ist an Gesetz und Recht gebunden, Art. 20 Abs. 2 Satz 1 und Abs. 3 GG.

Das zentrale Thema der grundgesetzlich gebotenen Unterscheidung zwischen Staat und Gesellschaft ist die Sicherung individueller Freiheit. Die Freiheit der Einzelnen wird dabei im Grundgesetz zweifach gesichert: durch die Möglichkeit, an der demokratischen Willensbildung mitzuwirken und die Begrenzung der Staatsgewalt gegenüber individueller Freiheit. Dabei ist es mit Blick auf den Begriff der Daseinsvorsorge entscheidend, dass sich die Frage nach der Freiheit des Einzelnen nicht ausschließlich im Sinne einer Freiheit vor staatlichen Eingriffen stellt. Teilweise verfügt die Einzelne nämlich nicht über die Voraussetzungen für eine freie und autonome Lebensgestaltung. Diese Voraussetzungen zu schaffen und zu erhalten, ist dann eine Aufgabe des Staats.

Mit Blick auf die Verortung der Daseinsvorsorge ist des Weiteren die wirtschaftspolitische Neutralität des Grundgesetzes von Bedeutung. Dieser Begriff sollte zwar nicht darüber hinwegtäuschen, dass die auf eine freie Entfaltung der Persönlichkeit angelegten Grundrechte einer liberalen Wirtschaftsverfassung nahestehen. Eine an den Grundrechten des Eigentums, der Berufsfreiheit, der Freizügigkeit und der allgemeinen Handlungsfreiheit orientierte Wirtschaftsverfassung wird - zumindest in wesentlichen Teilen - grundsätzlich wettbewerblich verfasst sein müssen. Freilich bedarf auch die wettbewerbsgetragene Wirtschaft der Ordnung und Korrektur. Die soziale Begleitung durch staatliche Eingriffe und Normen entspricht dabei dem Anliegen, dass die soziale Wirklichkeit nicht wesentlich hinter dem sozialen Staatsziel zurückbleiben darf. Die Bundesrepublik ist nämlich nicht nur ein demokratischer, sondern auch ein sozialer Rechtsstaat, Art. 20 Abs. 1 GG. Darin liegt keine unverbindliche, programmatisch-politische Aussage, sondern ein Rechtssatz: die Staatszielbestimmung, die alle Staatsorgane zur Verfolgung dieses sozialen Ziels verpflichtet.

Deswegen kann eine sozialstaatliche Interpretation der Grundrechte auch zu sozialen Verpflichtungen des Staates führen. Wenn zu den Aufgaben des Sozialstaates (auch) die Gewährleistung gleicher realer Freiheit gehört, kann eine sozialstaatliche Unterstützung geboten sein, wenn das Freiheitsrecht ansonsten leer läuft. Dies kann sich im Einzelfall sogar bis zu einem subjektiven Teilhabeoder Leistungsanspruch verdichten.

Genügen die Ergebnisse privater Initiative in als wesentlich definierten Bereichen nicht, um eine angemessene Versorgung der Bürgerinnen und Bürger zu sichern, gehört es deswegen zu den Staatsaufgaben, Leistungen der öffentlichen Hand zu gewährleisten oder selbst anzubieten. Bei den entsprechenden Maßnahmen ist jedoch zu unterscheiden zwischen 
- Leistungen an Bürger*innen, denen aufgrund ihrer finanziellen Leistungskraft der Ausschluss aus der Gesellschaft droht (Sozialleistung) und

- Leistungen an alle Bürger*innen, die unabhängig von dem persönlichen Leistungsvermögen erfolgen (Daseinsvorsorge) (Rüfner 2006, S. 1055).

Gemeinsam ist Sozialleistung und Daseinsvorsorge das Ziel, Not zu vermeiden und Güter und Dienstleistungen möglichst Vielen möglichst gleich zugänglich zu machen. Während der Schwerpunkt der Sozialpolitik darin liegt, Unterschiede im Zugang zu Dienstleistungen und Gütern auszugleichen, die in dem Sozialverhältnis begründet sind, zielt die Daseinsvorsorge auf Sicherheit und Allgemeinheit der Versorgung. Der sozialstaatliche Auftrag der Gleichheit macht den Begriff der Daseinsvorsorge deswegen nicht überflüssig.

Die Aufgabe der Daseinsvorsorge besteht selbstständig neben dem Prinzip des Sozialstaats. Sie folgt aus der Verpflichtung des Staats auf Gemeinwohl und Wohlfahrt und ist eine Reaktion auf die zunehmende Abhängigkeit eines jeden Menschen von fremden Leistungen. Aus Bevölkerungskonzentrationen, Arbeitsteilung sowie den Risiken der komplexen Produktions- und Verteilungsvorgänge folgen erhebliche Risiken und Herausforderungen für den Einzelnen. Anders als Sozialleistungen richtet sich die Daseinsvorsorge deshalb grundsätzlich an Jede*n (Knauff 2004, S. 48). Gekennzeichnet ist der Kreis dieser Leistungen dadurch, dass er um der einzelnen Bürgerin Willen angeboten wird (Rüfner 1996, S. 1044). Es geht nicht um Leistungen der Wirtschaftsförderung, nicht primär um Gewinnwirtschaft. Sie richten sich an die ,Normalbürger*innen', die an sich in der Lage sind, für ihr Auskommen selbst zu sorgen, allerdings einzelne wichtige Bedürfnisse weder aus eigener Kraft noch auf dem Markt befriedigen können (ebd.). Daseinsvorsorge erfolgt deswegen in der Regel nicht unentgeltlich, sondern mit dem Ziel der Kostendeckung.

Von zentraler Bedeutung ist dabei nicht, wer Leistungen der Daseinsvorsorge erbringt, sondern dass diese Leistungen nach am Gemeinwohl orientierten Grundsätzen erbracht werden (Knauff 2004, S. 48). Es wäre daher ein Missverständnis der Daseinsvorsorge, wenn diese im Sinne einer umfassenden Aufgabenübernahme durch staatliche Stellen verstanden werden würde. Ein staatliches Eingreifen ist erst dann erforderlich, wenn sich die gewünschte Gemeinwohlorientierung nicht ohne solche Eingriffe einstellt. Außerdem können sich staatliche Eingriffe auch darauf beschränken, der privaten Initiative sinnvolle Rahmenbedingungen zu setzen (Rüfner 2006, S. 1051). Erst wenn auch dies nicht ausreicht, ist eine staatliche Aufgabenübernahme geboten.

Vor diesem Hintergrund wird auch verständlich, warum sich der Staat zunehmend weniger als Leistungsstaat versteht, der die öffentlichen Aufgaben 
selbst erbringt. Vielmehr orientiert er sich zunehmend am Leitbild des Gewährleistungsstaats, der es Privaten im Rahmen hoheitlich gesetzter regulativer Vorgaben ermöglicht, Leistungen zu erbringen, die von wesentlicher Bedeutung für das Gemeinwohl sind. Dieses Leitbild ist aber gerade keine Abwendung von einem (modernen) Verständnis der Daseinsvorsorge, weil die damit verbundenen Ziele nicht relativiert werden. Es löst sich hingegen von der wenig treffenden (und nicht selten polemischen) Gegenüberstellung von privater Marktwirtschaft und öffentlicher Leistungserbringung.

Die konkreten Aufgaben der Daseinsvorsorge werden dann auf Grundlage der jeweils aktuellen Versorgungsbedürfnisse definiert und erbracht. Daraus folgt jedoch gerade keine Beliebigkeit: Die Sorge um eine preiswerte, sichere, flächendeckende und in der Tarifgestaltung Gemeinwohlbelange berücksichtigende Versorgung ist ein hinreichender Grund für eigene Einrichtungen der öffentlichen Hand, solange eine gleichwertige Versorgung durch Private nicht zu erwarten ist (Rüfner 2006, S. 1062). Die Daseinsvorsorge mit kommunalen Leistungen eigener Einrichtungen oder mittels der Gewährleistung ihrer Erbringung durch Private ist eine Rechtspflicht des an der Wohlfahrt der Bürger*innen orientierten Staats, festgehalten im Grundgesetz, Art. 28 Abs. 2 (Rüfner 2006, S. 1064).

Insoweit bleibt freilich zu beachten, dass Staatsaufgaben weiter gefasst sind als Rechtspflichten und unter den legitimen Staatsaufgaben darf der Staat wählen und Prioritäten setzen. Sie gehen nicht zuletzt auf die herrschenden gesellschaftlichen Leitbilder und die politischen Mehrheiten zurück (ebd.).

\section{$4 \quad$ Zur Karriere von Daseinsvorsorge und öffentlichem Verkehr in der Bundesrepublik Deutschland}

Als Forsthoff in den 1930er Jahren sein Konzept der Daseinsvorsorge entwarf, stand ihm auch der öffentliche Verkehr vor Augen. Allgemein zugängliche Mobilität war zu dieser Zeit noch nicht anders denkbar. Nach dem Zweiten Weltkrieg änderten sich die Voraussetzungen dafür grundlegend. Mit der Ausbreitung des MIV zu einem Massenphänomen konnte der gesellschaftlich notwendige Verkehr zu einem immer größeren Teil privat erbracht werden. Das staatlich gewährleistete Angebot kollektiver Verkehre wich zunehmend der Realisierung individueller Selbstbeweglichkeit im eigenen Pkw. Auf diese Weise eroberten sich die Bürgerinnen und Bürger den Verkehrssektor zurück und emanzipierten sich von obrigkeitsstaatlichen Vorgaben. Deshalb wird das Aufkommen des MIV 
nicht selten emphatisch als Akt individueller Befreiung und damit einhergehender Demokratisierung beschrieben (Burkart 1994).

Von diesem Zeitpunkt an entwickelten sich die zwei in ihrer Funktionsweise grundverschiedenen Verkehrssysteme weitgehend unabhängig voneinander. Während sich der MIV durch eine rasante Wachstumsdynamik auszeichnete, verblieb der öffentliche Verkehr weiterhin in der Sphäre staatlicher Leistungsverwaltung, die mit der rechtsethischen Konstruktion der Daseinsvorsorge legitimiert und (leider) nach zumeist paternalistischem Muster praktisch ausgelegt wurde. Wie sehr die inhaltliche Bestimmung der Daseinsvorsorge von den historischspezifischen gesellschaftspolitischen Rahmenbedingungen abhängt, verdeutlicht bereits die erste Krisendebatte über den öffentlichen Verkehr zu Beginn der 1970er Jahre. Dieser hatte damals schon deutlich an Attraktivität gegenüber dem MIV eingebüßt, was sich u. a. in immer höheren Subventionsleistungen der öffentlichen Hand niederschlug. Vor diesem Hintergrund entzündete sich eine lebhafte Diskussion über die Zukunft des öffentlichen Verkehrs. Dabei zeigen sich erstaunliche Parallelen zu aktuellen Auseinandersetzungen. Das gilt insbesondere für das Thema der ,Rationalisierung im Verkehr als Teil der Daseinsvorsorge", wie eine Tagung der Arbeitsgemeinschaft für Rationalisierung des Landes Nordrhein-Westfalen überschrieben war (AGR 1975). Während Rationalisierung im weitesten Sinne für vernunftgemäßes ökonomisches Handeln stand, wurde dieses Verständnis dem Prinzip der Daseinsvorsorge als am Gemeinwohl orientierte politische Rationalität gegenübergestellt. Dabei wurde erneut deutlich, dass der klaren Vorstellung von ökonomischer Rationalität mit einem diffusen Verständnis der Daseinsvorsorge nur unzulänglich begegnet wird.

In der Diskussion standen sich ähnliche Positionen wie heute gegenüber. Auf der einen Seite wurde die Warnung geäußert, ,dass auch der sogenannten Daseinsvorsorge des Staates Grenzen gesetzt sind“ (Wilkenloh 1975, S. 16). Auf der anderen Seite herrschte die Überzeugung, dass gerade im Verkehr ,die Grundinvestitionen im Wesentlichen vom Staat und den Kommunen durchgeführt werden“ müssen (Nehrling 1975, S. 17). Es bleibt festzuhalten, dass die genaue Ausgestaltung dessen, was Daseinsvorsorge zu leisten habe, auch damals aus dem Konzept nicht in erster Linie rechtlich abgeleitet wurde. Stattdessen sei Daseinsvorsorge ein politisch zu bestimmender Begriff. „Er bedarf meines Erachtens ebenso wie der Begriff der Lebensqualität der Ausfüllung durch die Politik, genauer gesagt, durch die Vorstellung der verschiedenen politischen gesellschaftlichen Gruppierungen, über die in einem Rechtsstaat wie dem unseren letztlich die gesetzgebenden Körperschaften, und nicht zuletzt die Körperschaften der Selbstverwaltung, zu entscheiden haben“ (Stukenberg 1975, S. 18). Die politische Ausgestaltung wurde schon damals der ökonomischen Rationalität nachgeordnet, 
denn sie hänge ,letzten Endes von der Machbarkeit ab, Machbarkeit hier einmal ganz brutal auf das Geld bezogen [Herv. d. Verf.]“ (Eichhoff 1975, S. 23). In der Begründungssemantik nimmt diese Position den heutigen Diskussionsstand vorweg.

Ein weiteres Beispiel ist die Diskussion auf Basis der Verordnung der Europäischen Union vom 26. Juli 2000 zur Liberalisierung des Personenverkehrs (KOM 2000) sowie der seinerzeitigen Spargesetze verschiedener Regierungen. Sie stellten grundsätzlich infrage, ob der öffentliche Verkehr ein Bestandteil der Daseinsvorsorge sei. Die Bezugnahme auf sie wirkt dabei häufig wie ein rotes Tuch im Konflikt zwischen Sozialstaatsbefürworterinnen und Vertretern einer freien Marktwirtschaft. Die Kritiker*innen bemängeln die Antiquiertheit dieses Rechtsbegriffs und fordern seine vollständige Abschaffung. An seine Stelle sollen staatliche Verantwortung für die Infrastruktur und eine Leistungserbringung nach privatwirtschaftlichen Kriterien treten. Das überholte Konzept sei schuld an der Ineffizienz und Servicefeindlichkeit der unter seinem Dach erbrachten Leistungen (Hermes 1998; Miegel 2003).

Die Gegenposition wird in erster Linie von Gewerkschaften und Sozialverbänden, aber auch dem Deutschen Städtetag vertreten (Deutscher Städtetag 2018, 2000). Sie argumentieren, dass im Rahmen der Daseinsvorsorge auch den sozial Benachteiligten, Alten, Kindern, Kranken - kurz allen, die auf die Solidarität in der Gesellschaft besonders angewiesen sind - lebensnotwendige Güter und Dienstleistungen bereitgestellt werden müssen, auch wenn sich diese nicht wirtschaftlich rechtfertigen lassen. Hier wird häufig der Begriff des Gemeinwohls verwendet. ${ }^{12}$ Außerdem sehen diese Akteure das Selbstverwaltungsrecht der Gemeinden bedroht (Deutscher Städtetag 2003). Die Diskussion um den öffentlichen Verkehr ist also eingebettet in einen allgemeinen Disput über die Daseinsvorsorge oder, um den Bogen noch weiter zu spannen, über die Frage, wie wenig Staat und wie viel Markt unsere Gesellschaft benötigt. Aus diesem Zusammenhang speist sich die spezielle Brisanz der Thematik. ${ }^{13}$ Vor diesem Hintergrund lässt sich die Auseinandersetzung um den öffentlichen Verkehr in der historischen Rückschau als spezifische Fallstudie eines großen Machtkampfs von Politik und Wirtschaft lesen (Acemoglu und Robinson 2013).

\footnotetext{
${ }^{12} \mathrm{Zu}$ dessen dem Begriff der Daseinsvorsorge verwandten Definitionsproblem Schuppert und Neidhardt (2002).

${ }^{13}$ Daher sind Beiträge, die das Thema differenziert behandeln, noch immer selten (z. B. Krautscheid 2009; Neu 2009).
} 
Die Folgen einer Herauslösung von Diensten aus der Daseinsvorsorge werden dann mitunter klar benannt: „Das Wettbewerbsgeschehen kann dazu führen, dass derartige Leistungen für bestimmte Regionen oder Bevölkerungsgruppen nicht in dem Umfang, der Struktur oder zu den Preisen erbracht werden, die die Politik für angemessen hält" (Schatz 2000, S. 10). Es ist also die Frage, ob die Politik die Daseinsvorsorge garantiert oder der Markt über sie entscheidet, ja sie im Zweifelsfall auflöst. „Letzteres würde die Integrationsleistung des Leviathan tangieren und zielt damit direkt auf die staatliche Legitimationsgrundlage“ (Becker 2002, S. 199). In Bezug auf den öffentlichen Verkehr stellt sich dies folgendermaßen dar: „Mobilität ist Gegenstand der privaten Lebensführung oder wirtschaftlicher Betätigung und kann als solche nicht der öffentlichen Bezuschussung unterliegen“ (FES 2001, S. 9). Im Ergebnis heißt das: Es gibt nur noch dort Verkehrsdienstleistungen, wo sie ihren Betreibern Gewinn einfahren. Ob es sich dabei dann noch um ,öffentlichen " Verkehr handelt, der sich dadurch auszeichnet, dass er für alle Menschen prinzipiell zugänglich ist, darf bezweifelt werden.

Demgegenüber hält das hier vertretene Konzept der Öffentlichen Mobilität an dem grundsätzlichen Anspruch des öffentlichen Verkehrs fest, die gesellschaftliche Teilhabe auch bei veränderten gesellschaftlichen Rahmenbedingungen durch ein angemessenes Verkehrsangebot zu gewährleisten. Dazu muss das Konzept der Daseinsvorsorge politisch neu ausbuchstabiert werden.

\section{Fazit und Ausblick}

Der öffentliche Personennahverkehr ist ein geradezu klassischer Fall der mit dem Begriff Daseinsvorsorge beschriebenen Aufgabe des Staates und seiner Verwaltung. Er spielt eine wesentliche Rolle für das Zusammenleben der Bürgerinnen und Bürger, ist jedoch in seiner Komplexität von der Einzelnen nicht selbstständig zu erbringen. Vielmehr bedarf es einer ordnenden Struktur, wie sie insbesondere auf Basis von Nahverkehrsplänen sinnvoll angelegt werden kann. Der Einzelne mag keinen originären Anspruch auf den Ausbau des öffentlichen Personennahverkehrs haben; gleichwohl gehört es zu den Aufgaben staatlicher Verwaltung, auf Basis des jeweiligen Lebensstandards und hier insbesondere der verkehrlichen Bedürfnisse und ihrer Auswirkung auf das Zusammenleben in Ballungszentren Verkehr und Mobilität sinnvoll zu gestalten.

Wenn das Konzept der Daseinsvorsorge im Verkehr zu Ende gedacht werden würde, läge es nahe, den Bürger*innen einen Hausanschluss für Mobilität zur Verfügung zu stellen (Schwedes und Daubitz 2012). Denn während andere Dienstleistungen und Waren der klassischen Daseinsvorsorge (Strom, Gas, Wasser, Telefonie und Internet) bis in nahezu jeden Haushalt hineinreichen, 
besteht das ÖPNV-Netz aus weiten Maschen. Man muss schließlich immer von Zuhause zur nächsten Bus-, Tram-, S- oder U-Bahnstation gelangen. Diese Wege können sehr weit sein. Dort angekommen, kann man mitunter sehr lange auf eine ,Beförderung' warten. Wenn umgestiegen werden muss, trifft dies auf den Anschluss ebenfalls häufig zu. Dieses Problem gilt es als öffentliche Herausforderung zu begreifen, sonst lösen die Menschen es weiterhin wie bisher vorzugsweise auf private Art und Weise: durch den Besitz eines Pkw mit allen für die Allgemeinheit daraus resultierenden negativen Folgen. Ging es früher darum, die allgemeine Zugänglichkeit von Mobilität zu gewährleisten, stellt sich heute die Aufgabe, die Konsequenzen der Verkehrsentwicklung in hochgradig mobilen Gesellschaften nachhaltig zu bewältigen. Damit stellt sich die Frage, ob dabei dem Prinzip der Daseinsvorsorge noch eine Relevanz zukommt.

Um ihm gerecht zu werden, können bestimmte politisch definierte Leistungen oder Leistungspakete zur exklusiven Bereitstellung durch einzelne Unternehmen ausgeschrieben und, wenn notwendig, subventioniert werden. Somit ist die Politik gefordert, festzulegen - zum Beispiel in Nahverkehrsplänen -, worin das allgemeine Interesse am öffentlichen Verkehr besteht und auf welche Weise ihre Vorgaben umgesetzt werden sollen.

Bei der Definition des allgemeinen Interesses an der verkehrlichen Gestaltung des öffentlichen Raums sollte, die Politik' nicht nur als Gesetzgeberin und ausführende Verwaltung, sondern vielmehr ,zivilgesellschaftlich ‘ verstanden werden. Die interessierte und betroffene Öffentlichkeit müsste z. B. in Bürger*innen- und Stadteilforen bei der Erstellung von regional differenzierten Nahverkehrsplänen und der Definition von ,ausreichender Verkehrsbedienung' stärker beteiligt werden, und zwar jenseits der häufig von lobbyistischen Interessenverbänden organisierten Alibiveranstaltung (Strasser 2004; Lorenz et al. 2020). Dieser Vorschlag legt nahe, sich an ähnlichen, aber durchaus zu optimierenden Verfahren bei Bebauungs- und Flächennutzungsplänen zu orientieren. Der Verwaltung (Länder, Landkreise und Kommunen) obliegt dann die Aufgabe, die verschiedenen regionalen und quartiersbezogenen Verkehrspläne miteinander abzustimmen. Der Erfolg des viel gepriesenen Schweizer Verkehrsmodells liegt nicht zuletzt in dessen basisdemokratischer Legitimation begründet (Breitenmoser et al. 2001; Sager et al. 1999).

Die prinzipielle Fragestellung, ob der öffentliche Verkehr die Daseinsvorsorge braucht, bzw. ob wir sie brauchen, muss in Anbetracht einer zunehmend differenzierten, verwobenen und für jede*n Einzelne*n zunehmend risikohaften Gesellschaft überraschen (Beck 1986). Unter solchen riskanten Bedingungen nimmt die Notwendigkeit einer überindividuellen, solidarischen Vorsorge für die Mehrheit der Bevölkerung kaum ab. Freilich ist der Vorsorgegedanke heute durch 
die - teilweise bewusste - Vermengung mit der Fürsorge als paternalistisches Prinzip diskreditiert. Er wird mittlerweile durch eine andere Begrifflichkeit ,moderner` zum Ausdruck gebracht, nämlich durch die Nachhaltigkeit. Während sich das Konzept der Daseinsvorsorge auf die vorausschauende Aufrechterhaltung der ökonomischen und sozialen Reproduktion beschränkte, beansprucht das Prinzip der Nachhaltigkeit außerdem die ökologischen Aspekte zukünftiger Gesellschaftsentwicklung zu berücksichtigen. Es überrascht daher nicht, dass sich die beiden Debatten auf verblüffende Weise ähneln. In beiden Fällen geht es einerseits um die gesellschaftspolitische Gewichtung kurzfristiger, betriebswirtschaftlicher Entscheidungen und andererseits um die Reflexion ihrer langfristigen sozialen und ökologischen Auswirkungen. So gesehen erscheint es nicht unwahrscheinlich, dass das Konzept der Daseinsvorsorge zukünftig im Nachhaltigkeitsdiskurs aufgehen wird.

Demgegenüber erinnert die Forderung nach einem grundsätzlichen Rückzug des Staats aus seiner Verantwortung für die gemeinwohlorientierte Vorsorge an das Verständnis des 19. Jh. Der damalige Nachtwächterstaat sah seine Aufgabe vor allem in der Aufrechterhaltung innerer und äußerer Sicherheit. Seine Funktion lag in der defensiven Haltung begründet, die formalen Freiheitsrechte jedes einzelnen Gesellschaftsmitglieds zu schützen, ohne jedoch selbst aktiv zu werden. Die materielle Grundlage der Existenz, die Vorsorge, blieb dem Schicksal jedes Einzelnen überantwortet. Die in den letzten Jahren wieder häufig vorgetragene Forderung, die Einzelne, ihr Eigentum und die Familie müssten gestärkt werden, mutet wie eine Renaissance der politischen Philosophie dieser Zeit an (Nida-Rümelin 2011).

Die Betonung individueller Einzelrechte wird auch im Diskurs um die Zukunft des öffentlichen Verkehrs immer wieder verwendet, z. B. mit der Aussage, dass er angesichts zunehmender Individualisierung nicht mehr zeitgemäß sei (Projektgruppe Mobilität 2001). Solche Argumentationsansätze überzeugen regelmäßig bereits deshalb nicht, weil sie persönliche Freiheit preisen, ohne nach deren Voraussetzungen zu fragen. Gerade dort, wo staatliches Handeln die persönliche Freiheit für alle Bürger*innen erst ermöglicht, erscheint der Gegensatz von Individuum und Staat kaum ausreichend, um die Komplexität der Aufgabe sachgerecht zu beschreiben.

Das Argument, Mobilität sei eine rein individuelle Angelegenheit und nicht von allgemeinem Interesse, kann zudem schnell mit dem Blick auf die im Autoverkehr zu beobachtende Massenindividualisierung ${ }^{14}$ entkräftet werden. Wo Mobilität eine

\footnotetext{
${ }^{14}$ Die Chiffre „MIV“ ließe sich auf diese Weise besser übersetzen.
} 
individuell erfahrbare (körperliche) Bewegung bezeichnet, tritt dem Einzelnen im Verkehr die Mobilität der anderen Individuen, der Allgemeinheit, als technisch vermitteltes System gegenüber. Diese dialektische Verschränkung von individueller Mobilität und allgemeinem Verkehrssystem wird deutlich im Begriffspaar Automobilität und öffentlicher Verkehr. Zwar verkehrt auch jede Autofahrerin öffentlich, doch wähnt sie sich selbst in ihrem Mobil. Wer ,öffentlich“ unterwegs ist, befindet sich dagegen in einem Verkehrsmittel. Diese Semantik signalisiert den scheinbaren Gegensatz von mobiler Selbstbestimmung versus fremdbestimmter Funktionalität. Wer Mobilität individuell zu lösen glaubt, blendet den Strukturzusammenhang des Verkehrs aus. ${ }^{15}$

Dass der Verkehr immer öffentlich geregelt wird - nur eben bislang vielfach im Sinne des MIV -, sollte ein gemeinsames Verständnis aller Verkehrs- und Gesellschaftsmitglieder sein. Dass dies häufig nicht der Fall ist, liegt vor allem daran, dass das Prinzip der Daseinsvorsorge bis heute auf den öffentlichen Verkehr beschränkt bleibt. Indem sich parallel zum öffentlichen Verkehr - mit seiner sozialen Verpflichtung, eine ausreichende Verkehrsbedienung für alle Gesellschaftsmitglieder zu gewährleisten - der von sozialen und ökologischen Verpflichtungen weitgehend unbelastete MIV etablierte, entstanden zwei grundverschiedene Verkehrssysteme, die sich ganz unterschiedlich gesellschaftlich legitimieren. Während der öffentliche Verkehr sich über seine Gemeinwohlorientierung im Rahmen der Daseinsvorsorge definiert, betont der MIV den individuellen Freiheitsgedanken.

Über die sich daraus ergebenden Zielkonflikte zwischen Allgemeinheit und Individuum wird in demokratischen Rechtssystemen politisch verhandelt. Seit dem Aufkommen des MIV als Konkurrenz zum ÖV wurde immer wieder auf die Notwendigkeit einer stärkeren Verknüpfung beider Verkehrssysteme hingewiesen. Schon früh wurde die Veröffentlichung des privaten Individualverkehrs sowie die Individualisierung des öffentlichen Kollektivverkehrs angestrebt. Auch heute zielt das allgemein anerkannte Leitbild einer Integrierten Verkehrsplanung auf die Verbindung der beiden unabhängigen Verkehrssysteme mit dem Ziel, die Verkehrsentwicklung nachhaltig am Gemeinwohl zu orientieren (siehe den Beitrag von Schwedes in diesem Band).

Dabei sei ergänzend darauf hingewiesen, dass die zunehmend relevante Diskussion um eine Verkehrswende auf mehr als den Ausbau des ÖPNV zielt - und

\footnotetext{
${ }^{15}$ „Der Einzelne und sein Selbstfahrzeug“ wäre der Titel der hier anschlussfähigen psychologischen Untersuchung von Automobilwerbung.
} 
damit auch das Verständnis des öffentlichen Verkehrs im Sinne einer Öffentlichen Mobilität erweitert. Der öffentliche Verkehr ist zwar das Rückgrat des Umweltverbundes und jeder umfassend verstandenen Verkehrswende. Gleichzeitig ist aber auch ein erheblicher Umbau der öffentlichen Infrastruktur geboten, der eine bislang stark am MIV angelegte Verteilung öffentlicher Flächen korrigiert und insbesondere die Interessen von zu Fuß Gehenden und Radfahrenden stärker in den Mittelpunkt rückt. Nicht zuletzt das Berliner Mobilitätsgesetz greift diesen Aspekt auf und enthält planerische Grundsätze für eine andere Verteilung öffentlicher Flächen zugunsten aller Verkehrsteilnehmenden (siehe den Beitrag von Kirchner in diesem Band).

Im Anschluss daran wird auch die Rolle neuer Mobilitätsangebote im Rahmen einer Öffentlichen Mobilität jenseits des MIV und des klassischen ÖPNV von Interesse sein (siehe den Beitrag von Wolking in diesem Band). Insbesondere Sharing-Angebote sind in ihrer Auswirkung auf den öffentlichen Raum und die Mobilität jeder Einzelnen immer noch in der Entwicklung begriffen. Wird jedoch der begrenzte öffentliche Raum und die Zunahme der Bevölkerung in vielen Ballungszentren berücksichtigt, so spricht viel für die Förderung von Mobilität durch geteilte Verkehrsmittel, um eine Neuverteilung öffentlicher Flächen umsetzen zu können. Auf Basis des Begriffs der Daseinsvorsorge und der damit verbundenen Aufgabe staatlicher Verwaltung liegt es nahe, dass auch der Aufbau solcher Systeme öffentlich-rechtlichen Grundsätzen unterliegen und dabei im Sinne eines flächendeckenden, preiswerten und diskriminierungsfreien Angebots gestaltet werden sollte (siehe den Beitrag von Daubitz in diesem Band). Sinnvoll verbunden können sich öffentlicher Nahverkehr und geteilte Mobilitätsangebote höchst effektiv und zum Nutzen aller zu einer Öffentlichen Mobilität ergänzen. Würden jedoch Angebote für Busse und Bahnen ebenso wie Angebote geteilter Verkehrsmittel allein nach marktwirtschaftlichen Prinzipien erfolgen, bliebe für eine geordnete, am Gemeinwohl orientierte verkehrspolitische Entwicklung wenig Raum.

\section{Literatur}

Acemoglu, Aron und J. A. Robinson. 2013. Warum Nationen scheitern. Die Ursprünge von Macht, Wohlstand und Armut. Frankfurt am Main: Fischer.

AGR - Arbeitsgemeinschaft für Rationalisierung des Landes Nordrhein-Westfalen, Hrsg. 1975. Rationalisierung im Verkehr als Teil der Daseinsvorsorge. Dortmund: Sander.

Ambrosius, Gerold. 1984. Der Staat als Unternehmer. Göttingen: Vandenhoeck \& Ruprecht. 
Ambrosius, Gerold. 1995. Kommunalwirtschaft im Spannungsfeld von Autonomisierung/ Privatisierung und Bindung/Regulierung (vom Ende des 19. Jh.s bis zu den 1930er Jahren). In Kommunalisierung im Spannungsfeld von Regulierung und Deregulierung im 19. und 20. Jh., Hrsg. J. Wysocki, 141-163. Berlin: Duncker \& Humblot.

Beck, Ulrich. 1986. Risikogesellschaft. Auf dem Weg in eine andere Moderne. Frankfurt am Main: Suhrkamp.

Becker, Hartmuth. 2002. Die Kategorie öffentlicher Güter als Grundlage von Staatstheorie und Staatswissenschaft. Berlin: Duncker \& Humblot.

Bendikat, Elfi. 1997. Öffentliche Verkehrssysteme im Spannungsfeld kommunaler Intervention im Metropolenvergleich: Berlin und Paris 1890-1914. In Mobilität für alle. Geschichte des öffentlichen Personennahverkehrs in der Stadt zwischen technischem Fortschritt und sozialer Pflicht, Hrsg. H.-L. Dienel und B. Schmucki, 149-182. Stuttgart: Franz Steiner.

Brandt, Jürgen. 1929. Die wirtschaftliche Betätigung der öffentlichen Hand. Jena: Fischer.

Breitenmoser, Bernadette, R. Furrer, L. Raymann. 2001. Wege zur Nachhaltigkeit im Verkehr. Konsens auf lokaler Ebene ist Voraussetzung. Neue Zürcher Zeitung, 3. Oktober 2001.

Burkart, Günter. 1994. Individuelle Mobilität und soziale Integration. Zur Soziologie des Automobilismus. In Soziale Welt, Heft 2: 216-241.

Dessauer, Friedrich. 1928. Philosophie der Technik. Das Problem der Realisierung. 2. Aufl. Bonn: Friedrich Cohen.

Deutscher Städtetag. 2000. Europäische Wettbewerbspolitik gefährdet kommunale Daseinsvorsorge. Pressedienst des Deutschen Städtetags vom 3. Mai 2000. Berlin.

Deutscher Städtetag. 2003. Die Stadt der Zukunft. Beschluss der 32. ordentlichen Hauptversammlung des Deutschen Städtetages am 15. Mai 2003 in Mannheim. Dokumentiert in Schader-Stiftung (2003): 310-316. Darmstadt.

Deutscher Städtetag, Hrsg. 2018. Nachhaltige städtische Mobilität für alle - Agenda für eine Verkehrswende aus kommunaler Sicht. Berlin und Köln: Deutscher Städtetag. http:// www.staedtetag.de/imperia/md/content/dst/veroeffentlichungen/mat/positionspapiernachhaltige-staedtische-mobilitaet.pdf (31.12.2020).

Eichhoff, Eugen. 1975. Redebeitrag auf der Podiums- und Generaldiskussion der Arbeitsgemeinschaft für Rationalisierung des Landes Nordrhein-Westfalen. In Rationalisierung im Verkehr als Teil der Daseinsvorsorge, Hrsg. AGR - Arbeitsgemeinschaft für Rationalisierung des Landes Nordrhein-Westfalen, 15-36. Dortmund: Sander.

Fisch, Stefan. 1997. Nahverkehr aus der Sicht der Stadtplanungsgeschichte. Anmerkungen eines Verwaltungs- und Planungshistorikers. In Mobilität für alle. Geschichte des öffentlichen Personennahverkehrs in der Stadt zwischen technischem Fortschritt und sozialer Pflicht, Hrsg. H.-L. Dienel und B. Schmucki, 51-61. Stuttgart: Franz Steiner.

Forsthoff, Ernst. 1933. Der totale Staat. Hamburg: Hanseatische Verlagsanstalt.

Forsthoff, Ernst. 1938. Die Verwaltung als Leistungsträger. Stuttgart/Berlin: Kohlhammer.

Forsthoff, Ernst. 1950. Lehrbuch des Verwaltungsrechts. Band 1. Allgemeiner Teil. München/Berlin: C. H. Beck.

Gerber, Walter. 1928. Die öffentliche Unternehmung in privatrechtlicher Form. Zürich: Girsberger.

Grimm, Dieter. 1993. Der Staat in der kontinentaleuropäischen Tradition. In Abschied vom Staat - Rückkehr zum Staat?, Hrsg. R. Voigt, 27-50. Baden-Baden: Nomos. 
Gröttrup, Hendrik. 1973. Die kommunale Leistungsverwaltung. Stuttgart: Kohlhammer.

Günther, Frieder. 2011. Ordnen, gestalten, bewahren. Radikales Ordnungsdenken von deutschen Rechtsintellektuellen der Rechtswissenschaft 1920 bis 1960. In: Vierteljahrshefte für Zeitgeschichte, Heft 3, 353-384.

Hermes, Georg. 1998. Staatliche Infrastrukturverantwortung. Rechtliche Grundstrukturen netzgebundener Transport- und Übertragungssysteme zwischen Daseinsvorsorge und Wettbewerbsregulierung am Beispiel der leitungsgebundenen Energieversorgung in Europa. Tübingen: Mohr Siebeck.

Jaspers, Karl. 1931 [1971]. Die geistige Situation der Zeit. 7. Abdruck der im Sommer 1932 bearbeiteten 5. Aufl. Berlin: de Gruyter.

Krautscheid, Andreas, Hrsg. 2009. Die Daseinsvorsorge im Spannungsfeld von europäischem Wettbewerb und Gemeinwohl. Eine sektorspezifische Betrachtung. Wiesbaden: Springer VS.

Knauff, Matthias. 2004. Der Gewährleistungsstaat: Reform der Daseinsvorsorge. Berlin: Duncker \& Humblot.

Kocka, Jürgen. 1999. Klassengesellschaft im Krieg. Deutsche Sozialgeschichte 1914-1918. 2. durchgesehene und ergänzte Aufl. Göttingen: Vandenhoeck \& Ruprecht.

KOM - Kommission der Europäischen Gemeinschaften. 2000. Verordnung des Europäischen Parlaments und des Rates über die Maßnahmen der Mitgliederstaaten im Zusammenhang mit Anforderungen des öffentlichen Dienstes und der Vergabe öffentlicher Dienstleistungsaufträge für den Personenverkehr auf der Schiene, Straße und Binnenschiffahrtswegen. Kom (2000) 7 vom 26. Juli 2000. Brüssel.

Koselleck, Reinhard. 1987. Preußen zwischen Reform und Revolution. Allgemeines Landrecht, Verwaltung und soziale Bewegung von 1791 bis 1848. Stuttgart: Klett-Cotta.

Lederer, Emil. 1979. Kapitalismus, Klassenstruktur und Probleme der Demokratie in Deutschland 1910-1940. Buchreihe Kritische Studien zur Geschichtswissenschaft, Bd. 39, Hrsg. J. Kocka. Göttingen: Vandenhoeck \& Ruprecht.

Lessenich, Stephan. 2000. Soziologische Erklärungsansätze zu Entstehung und Funktion des Sozialstaats. In Soziologie des Sozialstaates. Gesellschaftliche Grundlagen, historische Zusammenhänge und aktuelle Entwicklungstendenzen, Hrsg. J. Allmendinger und W. Ludwig-Mayerhofer, 39-78. Weinheim: Beltz.

Lorenz, Astrid, C. P. Hoffmann und U. Hitschfeld, Hrsg. 2020. Partizipation für alle und alles? Fallstricke, Grenzen und Möglichkeiten. Wiesbaden: Springer VS.

FES - Managerkreis der Friedrich-Ebert-Stiftung. 2001. Verkehrspolitik im Strukturwandel: die neuen Chancen der Mobilität. Thesenpapier. Federführung: F. Mietsch. Berlin. https://library.fes.de/fulltext/managerkreis/01130.htm. Zugriff: 01.07.2020.

Meinel, Florian. 2011. Der Jurist in der industriellen Gesellschaft. Ernst Forsthoff und seine Zeit. Berlin: de Gruyter.

Miegel, Meinhard. 2003. Die Zukunft der öffentlichen Daseinsvorsorge. Hrsg. SchaderStiftung (2003): 28-37. Darmstadt.

Niebuhr, Heinrich. 1928. Öffentliche Unternehmungen und Privatwirtschaft. Leipzig: G. A. Gloeckner.

Nehrling, Heinz. 1975. Redebeitrag auf der Podiums- und Generaldiskussion der Arbeitsgemeinschaft für Rationalisierung des Landes Nordrhein-Westfalen. In Rationalisierung im Verkehr als Teil der Daseinsvorsorge, Hrsg. AGR - Arbeitsgemeinschaft für Rationalisierung des Landes Nordrhein-Westfalen, 15-36. Dortmund: Sander. 
Neu, Claudia, Hrsg. 2009. Daseinsvorsorge. Eine gesellschaftswissenschaftliche Annäherung. Wiesbaden: Springer VS.

Nida-Rümelin, Julian. 2011. Die Optimierungsfalle. Philosophie einer humanen Ökonomie. München: Irisiana.

Niederich, Nikolaus. 1997. Nahverkehrsbetriebe als Wirtschaftsunternehmen: Die Stuttgarter Straßenbahnen und ihre Eigentümer von 1868 bis 1918. In Mobilität für alle. Geschichte des öffentlichen Personennahverkehrs in der Stadt zwischen technischem Fortschritt und sozialer Pflicht, Hrsg. H.-L. Dienel und B. Schmucki, 83-108. Stuttgart: Franz Steiner.

Projektgruppe Mobilität. 2001. Kurswechsel im öffentlichen Verkehr. Mit automobilen Angeboten in den Wettbewerb. Berlin: edition sigma.

Reulecke, Jürgen. 1985. Geschichte der Urbanisierung in Deutschland. Frankfurt am Main: suhrkamp.

Ringwald, Roman. 2008. Daseinsvorsorge als Rechtsbegriff. Forsthoff, Grundgesetz und Grundversorgung. Frankfurt M.: Peter Lang.

Ritter, Gerhard A. 1991. Der Sozialstaat. Entstehung und Entwicklung im internationalen Vergleich. München: de Gruyter Oldenbourg.

Ronellenfitsch, Michael. 2003. Daseinsvorsorge als Rechtsbegriff - Aktuelle Entwicklungen im nationalen und Europäischen Recht. In Ernst Forsthoff: Kolloquium aus Anlass des 100. Geburtstags von Prof. Dr. Dr. h. c. Ernst Forsthoff, Hrsg. W. Blümel. 53-114. Berlin: Duncker \& Humblot.

Rüfner, Wolfgang. 1996. Daseinsvorsorge und soziale Sicherheit. In Handbuch des Staatsrechts, Hrsg. J. Isensee, P. Kirchhof, 2. Auflage, Band 3, 1037-1085. Heidelberg: C.F. Müller.

Rüfner, Wolfgang. 2006. Daseinsvorsorge und soziale Sicherheit. In Handbuch des Staatsrechts, Hrsg. J. Isensee, P. Kirchhof, 2. Auflage, Band 3, 1049-1107. Heidelberg: C.F. Müller.

Sager, Fritz, V. Kaufmann und D. Joye. 1999. Die Koordination von Raumplanung und Verkehrspolitik in urbanen Räumen der Schweiz: Determinanten der politischen Geographie, der politischen Kultur oder der institutionellen Struktur. In: Swiss Political Science Review, Heft 5: 25-55.

Saitzew, Manuel. 1930. Die öffentliche Unternehmung der Gegenwart. Tübingen: Mohr.

Schatz, Klaus-Werner. 2000. Die öffentliche Daseinsvorsorge im Spannungsfeld des EUWettbewerbs. Co-Referat anlässlich des 1. Brüsseler Gesprächs mit dem Thema „Die öffentliche Daseinsvorsorge im Spannungsfeld des EU-Wettbewerbsrechts“ der SPD Brüssel am 20.09.2000.

Scheuner, Ulrich. 1978 [1969]. Das Gesetz als Auftrag der Verwaltung. In Staatstheorie und Staatsrecht, Hrsg. J. W. Rüfner, 545-565. Berlin: Kohlhammer.

Schuppert, Gunnar F. und F. Neidhardt, Hrsg. 2002. Gemeinwohl - Auf der Suche nach Substanz. WZB-Jahrbuch 2002. Berlin: edition sigma.

Schmidt, Manfred G. 2005. Der deutsche Sozialstaat. Geschichte und Gegenwart. München: C. H. Beck.

Schwedes, Oliver und S. Daubitz. 2012. Hausanschluss Mobilität. Erfahrungen und Potenziale von Erreichbarkeitsplanung. Hrsg. Verbraucherzentrale Bundesverband. Berlin. https://www.vzbv.de/sites/default/files/downloads/Hausanschluss_Mobilitaet_ vzbv_2012.pdf. Zugriff: 25.04.2020. 
Smith, Adam. 1996 [1789]. Der Wohlstand der Nationen. Eine Untersuchung seiner Natur und seiner Ursachen. München: dtv.

Strasser, Karsten. 2004. Der Öffentliche Straßenpersonenverkehr im Umbruch. Was passiert auf dem Weg von der kommunalen Eigenproduktion zum Ausschreibungswettbewerb? In Wir können auch anders. Perspektiven von Demokratie und Partizipation, Hrsg. N. Fröhler, S. Hürtgen, C. Schlüter und M. Thiedke, 294-309. Münster.

Stukenberg, Helmut. 1975. Redebeitrag auf der Podiums- und Generaldiskussion der Arbeitsgemeinschaft für Rationalisierung des Landes Nordrhein-Westfalen. In Rationalisierung im Verkehr als Teil der Daseinsvorsorge, Hrsg. AGR - Arbeitsgemeinschaft für Rationalisierung des Landes Nordrhein-Westfalen, 15-36. Dortmund: Sander.

Wagner, Peter. 1995. Soziologie der Moderne. Freiheit und Disziplin. Frankfurt am Main/ New York: Campus.

Wehler, Hans Ulrich. 1995. Deutsche Gesellschaftsgeschichte. Bd. 3, Von der,Deutschen Doppelrevolution ' bis zum Beginn des Ersten Weltkrieges. München: C. H. Beck.

Wehler, Hans-Ulrich. 2014. Die Deutschen und der Kapitalismus. Essays zur Geschichte. München: Beck.

Wiedenfeld, Kurt. 1927. Die Wirtschaft der öffentlichen Hand. In Grundriß der Betriebswirtschaftslehre. Band 2, Hrsg. W. Mahlberg, E. Schmalenbach, F. Schmidt und E. Walb, 45-62. Leipzig: Gloeckner.

Wilkenloh, Friedhelm. 1975. Redebeitrag auf der Podiums- und Generaldiskussion der Arbeitsgemeinschaft für Rationalisierung des Landes Nordrhein-Westfalen. In Rationalisierung im Verkehr als Teil der Daseinsvorsorge, Hrsg. AGR - Arbeitsgemeinschaft für Rationalisierung des Landes Nordrhein-Westfalen, 15-36. Dortmund: Sander.

Wolf, Winfried. 1992. Eisenbahn und Autowahn. Personen- und Gütertransport auf Schiene und Straße. Geschichte, Bilanz, Perspektive. Hamburg: Rasch und Röhring.

Wysocki, Josef, Hrsg. 1995. Kommunalisierung im Spannungsfeld von Regulierung und Deregulierung im 19. und 20. Jh. Berlin: Duncker \& Humblot.

Open Access Dieses Kapitel wird unter der Creative Commons Namensnennung 4.0 International Lizenz (http://creativecommons.org/licenses/by/4.0/deed.de) veröffentlicht, welche die Nutzung, Vervielfältigung, Bearbeitung, Verbreitung und Wiedergabe in jeglichem Medium und Format erlaubt, sofern Sie den/die ursprünglichen Autor(en) und die Quelle ordnungsgemäß nennen, einen Link zur Creative Commons Lizenz beifügen und angeben, ob Änderungen vorgenommen wurden.

Die in diesem Kapitel enthaltenen Bilder und sonstiges Drittmaterial unterliegen ebenfalls der genannten Creative Commons Lizenz, sofern sich aus der Abbildungslegende nichts anderes ergibt. Sofern das betreffende Material nicht unter der genannten Creative Commons Lizenz steht und die betreffende Handlung nicht nach gesetzlichen Vorschriften erlaubt ist, ist für die oben aufgeführten Weiterverwendungen des Materials die Einwilligung des jeweiligen Rechteinhabers einzuholen.

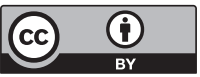

